\title{
Soins des patients atteints d'une mucoviscidose
}

\author{
Traitement, dépistage et évolution clinique
}

\section{Birgitta Strandvik}

Service de Pédiatrie, Institut des Sciences Cliniques, Académie Sahlgrenska, Université de Göteborg, Göteborg, Suède

\section{Mots-clés}

Nutrition - Acides gras essentiels · Staphylococcus aureus • Pseudomonas aeruginosa - Traitement intraveineux à domicile $\cdot$ Transplantation - Dépistage neonatal · Taux de survie

\section{Résumé}

La mucoviscidose, la maladie pédiatrique autosomique héréditaire la plus fréquente, n'atteint plus uniquement des enfants, car son traitement moderne a permis aux patients de vivre plus longtemps avec une meilleure qualité de vie. Voici plus de 16 ans, l'identification du gène responsable et de la mutation la plus fréquente, $\Delta \mathrm{F} 508$, a suscité l'espoir général d'une guérison rapide de cette maladie. Ces découvertes ont motivé une forte intensification des recherches sur la physiopathologie, mais, à ce jour, les améliorations de la prise en charge clinique ont été totalement dues au développement des traitements traditionnels. L'amélioration de la situation des patients est liée à une meilleure supplémentation en enzymes pancréatiques et à un traitement nutritionnel plus intensif, en prêtant une attention particulière aux anomalies du métabolisme des lipides. Le traitement respiratoire a permis de mieux préserver la fonction respiratoire des patients adultes, grâce à l'accès à un ensemble d'antibiotiques efficaces et au développement de nouvelles strategies, à des mucolytiques ainsi que des incitations à l'activité physique dès le plus jeune âge en association à des modifications de la kinésithérapie et à l'amélioration des résultats de la trans- plantation pulmonaire en tant que traitement de dernier recours. Une atteinte hépatique est plus souvent reconnue et plus fréquemment traitée à long terme par l'acide ursodésoxycholique, bien que l'effet préventif de ce produit vis-àvis de la progression de l'hépatopathie n'ait pas été totalement évalué. Le dépistage néonatal est de plus en plus fréquent, ce qui suscite de nouveaux problèmes à court terme, tels ceux de nature psychologique liés à la détection de porteurs ou de sujets atteints d'une forme très peu sévère, et tels que l'évaluation des résultats à long terme afin de démontrer que le statut clinique s'améliore et que la survie augmente encore. Les limitations de survie encore observées semblent principalement dues au mystère sous-tendant la colonisation chronique des voies aériennes par des micro-organismes.

Copyright $\odot 2006$ Nestec Ltd., Vevey/S. Karger AG, Basel

\section{Introduction}

A l'époque de l'identification de la mucoviscidose comme affection distincte de la maladie coeliaque en 1936-1938 [1,2], le traitement était très limité et, en conséquence, la mortalité demeurait élevée au cours de la première année de la vie [3]. Bien que le premier patient diagnostiqué à l'âge adulte ait été décrit en 1960 [4], la connaissance de cette maladie par les médecins est encore largement insuffisante, et des cas peu sévères peuvent donc ne pas être reconnus, peut-être même pendant

\begin{tabular}{ll}
\hline KARGER & (c) 2006 Nestec Ltd., Vevey/S. Karger AG, Basel \\
0250-9644/06/0643-0131\$23.50/0 \\
$\begin{array}{l}\text { Fax +4161306 1234 } \\
\begin{array}{l}\text { E-Mail karger@karger.ch } \\
\text { www.karger.com }\end{array}\end{array}$ & $\begin{array}{l}\text { Accessible en ligne à: } \\
\text { www.karger.com/anf }\end{array}$
\end{tabular}


toute la durée de la vie du sujet. Le traitement de la mucoviscidose pose le problème que les cas très discrets peuvent également se détériorer rapidement après une infection virale ou en raison d'autres facteurs encore non reconnus, ce qui implique que, quand le diagnostic a été porté, il est important de se préparer à traiter des troubles peu sévères. Ces circonstances ont justifié le dépistage néonatal, bien qu'aucun traitement curatif ne soit encore disponible. Les premiers traitements par supplémentation en enzymes pancréatiques n'étaient pas d'une efficacité satisfaisante et les troubles nutritionnels étaient importants. De ce fait, la mortalité infantile était importante et les gastro-entérologues pédiatres assuraient les principaux soins des patients mucoviscidosiques. Le développement de supplémentations en enzymes pancréatiques encapsulés dans des microsphères gastrorésistantes a entraîné une amélioration de leur efficacité, une meilleure absorption des nutriments et une amélioration de la survie précoce, et les troubles respiratoires ont donc fait l'objet d'une plus grande attention. Au cours des années 1970, la disponibilité de plusieurs nouvelles $\beta$-lactamines a permis de cibler davantage l'atteinte respiratoire. Avec la poursuite du développement des traitements de cette atteinte, les soins primaires ont été de plus en plus assurés par des pneumologues. L'amélioration des résultats de la transplantation pulmonaire déplacera à nouveau le centre d'intérêt sur des complications digestives telles que l'atteinte hépatobiliaire, mais également sur d'autres problèmes liés à la mucoviscidose, par exemple les complications rénales, la fragilité osseuse, le diabète et les troubles de la fertilité. En retour, la nécessité de centraliser les soins des patients mucoviscidosiques autour d'une équipe de professionnels apparaitra clairement, tant en ce qui concerne les soins médicaux que paramedicaux. Alors que les patients vivent plus longtemps et sont en meilleure situation pour avoir une vie sociale normale, les stratégies thérapeutiques doivent être adaptées afin d'améliorer l'observance du traitement, ce qui confronte les professionnels de santé à de nouveaux défis dans les soins des patients atteints de mucoviscidose.

\section{Organisation des soins des patients atteints d'une mucoviscidose}

Dès les années 1960, il était reconnu que les soins centralisés des patients souffrant de mucoviscidose étaient à l'origine d'une meilleure survie, et qu'une étroite coopération entre les centres de traitement de cette maladie et les médecins locaux intéressés par les problèmes qu'elle pose pouvait également contribuer à améliorer le traitement, dans la mesure où les patients étaient examinés au moins une fois par an au centre [5]. Les motifs en sont les nuances des troubles initiaux, dont la reconnaissance nécessite une longue expérience du médecin pour un traitement optimal. Cette situation implique également qu'un centre de soins de la mucoviscidose ne soit pas trop petit, donc traite plus de 50 patients, et ne soit sans doute pas trop grand non plus car la connaissance personnelle de chaque patient facilite l'évaluation correcte du statut actuel et de la nécessité éventuelle d'un traitement plus intensif. Il est établi que la survie et l'état général des patients sont meilleurs s'ils sont examinés une fois par an dans le cadre d'un bilan par rapport à des visites moins fréquentes. Il est également bien reconnu de nos jours qu'un centre doit être organisé sous la forme d'une équipe se composant d'un médecin, d'une infirmière ou d'un infirmier, d'un kinésithérapeute, d'un diététicien, d'un travailleur social, d'un psychologue, d'une secrétaire et d'un assistant technique pour la réalisation du test de la sueur [6]. Cette équipe doit également coopérer avec des experts tels que des généticiens ainsi qu’avec une unité de bactériologie intéressée par les problèmes spécifiques de cultures de l'expectoration chez les patients mucoviscidosiques, une unité de physiologie clinique intéressée par les explorations fonctionnelles respiratoires, une unité de radiologie, etc. Quelques centres de traitement de la mucoviscidose doivent également œuvrer en étroite collaboration avec des unités effectuant des transplantations pulmonaires et hépatiques.

\section{Besoins en énergie}

Il est recommandé que les apports énergétiques soient de $120 \%$ chez les patients atteints de mucoviscidose par rapport aux témoins en bonne santé, en raison de l'augmentation du métabolisme basal [7]. La dépense totale d'énergie diffère en fonction de l'activité physique et de l'altération de la fonction respiratoire, mais les résultats sont contradictoires quant à une association avec le génotype $[8,9]$. La croissance de certains patients est adéquate sans supplémentation énergétique [10], bien que la plupart des études aient montré que, en règle générale, il était difficile de satisfaire même les apports énergétiques recommandés chez les patients atteints d'une mucoviscidose, ce qui pourrait contribuer aux difficultés d'obtention d'une croissance normale [11]. Dans ce contexte, il faut noter que les patients porteurs de mutations telles que $\Delta \mathrm{F} 508$, considérées comme associées à des besoins 
énergétiques élevés, présentent également une insuffisance pancréatique et semblent plus sujets à la survenue d'une carence en acides gras essentiels [12]. Chez le rat, une carence en acides gras essentiels est associée à une augmentation des dépenses énergétiques.

Afin d'obtenir des apports énergétiques élevés, ces patients doivent recevoir un régime riche en énergie, où les lipides contribuent à $40-45 \%$ de l'énergie (E\%) comparativement à, au maximum, $30 \mathrm{E} \%$ chez les adultes en bonne santé, mais les apports en lipides n'atteignent cette valeur élevée que chez quelques patients [11]. Il est toutefois nécessaire de corriger la faible concentration plasmatique bien documentée de l'acide linoléique (18:2n-6). De ce fait, des acides gras polyinsaturés doivent contribuer à 10-15 E\% de l'apport énergétique. Un apport élevé en acides gras n- 6 normalise habituellement la faible concentration sérique de l'acide docosahéxanoïque $[12,13]$. Les patients mucoviscidosiques bénéficient d'une augmentation des apports en acides gras n-3, car les eicosanoïdes provenant de l'acide eicosapentaénoïque (20:5n-3), la prostaglandine $\mathrm{E}_{3}$ et la série des leucotriènes- 5 sont antiinflammatoires et peuvent compenser l'augmentation de la production d'eicosanoïdes à partir de la cascade de l'acide arachidonique (20:4n-6), qui est augmentée dans la mucoviscidose $[14,15]$, et dont la plupart des éléments stimulent l'inflammation.

Une augmentation de l'activité physique, dans le cadre du traitement moderne, accroît les besoins énergétiques. Des exacerbations respiratoires, associées à une augmentation de la fréquence respiratoire et à l'impact général d'une infection accroissent encore les besoins supplémentaires en énergie, d'autant plus que l'état du patient est altéré. Simultanément, l'augmentation des cytokines due à l'infection peut altérer l'appétit, ce qui transforme souvent les repas en problèmes chez les patients atteints d'une mucoviscidose [16]. Cette situation nécessite de l'ingéniosité afin de stimuler la consommation d'aliments et de prévenir ainsi une déviation vers le catabolisme, situation qui peut rapidement demander des mesures invasives telles qu'une nutrition entérale.

La prise de suppléments commerciaux à titre de collations peut augmenter les apports énergétiques mais n'est pas bénéfique si elle est effectuée afin de réduire les repas habituels, car la teneur de ces suppléments en glucides est comparativement élevée et les acides gras à chaîne moyenne (triglycérides à chaîne moyenne) ne sont pas des substituts de l'acide linoléique. Chez le jeune enfant, l'exacerbation d'une infection respiratoire peut temporairement altérer la fonction pancréatique résiduelle et l'apparition d'une diarrhée ou d'autres troubles digestifs peut être un symptôme précoce, indiquant la nécessité d'un traitement plus intensif de l'insuffisance pancréatique et de l'infection respiratoire.

Même avec le traitement par supplémentation en enzymes pancréatiques actuellement disponible, il est très souvent difficile de normaliser l'absorption de lipides, tant en état stable que lors d'une exacerbation, ce qui indique la nécessité d'améliorer encore ce traitement, et montre également que d'autres facteurs interviennent dans l'altération de l'absorption des lipides [17]. De nouvelles formes de supplémentation en enzymes pancréatiques par des lipases bactériennes et une lipase humaine stimulée par les sels biliaires recombinante sont étudiées dans le cadre d'essais cliniques $[18,19]$. Une atteinte hépatique sévère est rare et, de ce fait, la faible synthèse d'acides biliaires contribue probablement peu à l'altération de la lipolyse [20]. Toutefois, des complications biliaires peuvent diminuer le flux de bile et donc contribuer à une faible concentration intraluminale en sels biliaires et à la malabsorption [21]. Un autre facteur important pourrait être qu'une faible concentration plasmatique d'acide linoléique, indiquant une carence en acides gras essentiels, puisse altérer l'absorption des lipides en diminuant le transport transcellulaire de ceux-ci [22]. En raison de l'anomalie de l'absorption induite par la carence en acides gras essentiels (faible concentration d'acide linoléique), un apport d'acide linoléique par voie intraveineuse pourrait même être nécessaire en cas de carence sévère [23]. Les patients porteurs de mutations liées à un phénotype sévère sont plus sujets à avoir une faible concentration plasmatique d'acide linoléique et pourraient nécessiter plus souvent une supplémentation [12]. D’un point de vue pratique, il pourrait être recommandé d'utiliser l'abord intraveineux au cours des trois derniers jours du traitement d'une exacerbation respiratoire par antibiotique pour administrer $10 \mathrm{ml}$ d'Intralipid à $10 \%$ (ou de la quantité correspondante d'une émulsion comparable)/ $\mathrm{kg}$ de poids par jour pendant $6-8$ heures. Il est important que la perfusion ne soit pas trop rapide et de ne pas la réaliser à la phase aiguë de l'infection, car l'activité de la lipoprotéine lipase peut être réduite. Dans ce contexte, des dispositifs spéciaux pour perfusion des émulsions sont préférables. Chez les patients présentant une carence sévère en acides gras essentiels, un traitement similaire peut être recommandé pendant 3 jours toutes les $2-3$ semaines pendant 4-6 mois afin de normaliser la concentration sérique de l'acide linoléique, avec une amélioration simultanée des fonctions hépatiques et rénales [24, 25].

Chez les patients présentant déjà une malnutrition, habituellement en association à une altération sévère de la 
Tableau 1. Apport nutritionnel quotidien recommandé chez les patients atteints d'une mucoviscidose $\mathrm{e}^{1}$

\begin{tabular}{lll}
\hline Nutriment & Recommandation & Remarques \\
\hline Énergie & $120 \%$ de l'AQR ${ }^{2}$ & $\begin{array}{l}\text { Certains patients porteurs de mutations ne con- } \\
\text { férant pas une forme sévère ne nécessitent pas des } \\
\text { apports supplémentaires en énergie. Les apports en } \\
\end{array}$ \\
& $\begin{array}{l}\text { énergie doivent être accrus chez tout patient au } \\
\text { cours d'une infection. }\end{array}$ \\
\hline
\end{tabular}

\begin{tabular}{|c|c|c|}
\hline \multicolumn{3}{|l|}{ Macronutriments } \\
\hline Protéines & $15 \mathrm{E} \%$ & \\
\hline & 10-15 E\% sous forme d'acides gras polyinsaturés & $\begin{array}{l}\text { Huile de maïs, de soja ou de colza de raisin, } \\
10-15 \mathrm{ml} / \text { jour }^{3}\end{array}$ \\
\hline Glucides & $40-45 \mathrm{E} \%$ & $<10 \%$ énergie sous forme de saccharose \\
\hline \multicolumn{3}{|l|}{ Micronutriments } \\
\hline$\beta$-carotène & $0,5-1 \mathrm{mg} / \mathrm{kg}$ & \\
\hline D-calciférol & 400-800 UI $(10-20 \mu \mathrm{g})$ & \\
\hline E $\alpha$-tocophérol & $200-600 \mathrm{mg}$ & \\
\hline K (phytoménadione) & 5-10 mg par voie intramusculaire avant chirurgie & Mauvaise absorption intestinale \\
\hline \multicolumn{2}{|l|}{ Vitamines B } & Habituellement apportées par l'alimentation \\
\hline Vitamine $\mathrm{B}_{6}$ & $40-80 \mathrm{mg} /$ jour & $\mathrm{B}_{6}$ peut être indiquée \\
\hline Calcium & $\begin{array}{l}\text { Identique à une population en bonne santé du } \\
\text { même âge }\end{array}$ & $\begin{array}{l}\text { Pour des informations supplémentaires voir } \\
\text { réf. } 28,29\end{array}$ \\
\hline \multicolumn{3}{|c|}{$\begin{array}{l}{ }^{1} \text { La plupart des patients nécessitent des apports supplémentaires en lipides et en vitamins liposolubles comparativement au restant } \\
\text { la famille. } \\
{ }^{2} \text { Apport quotidien recommandé pour la population en bonne santé. }\end{array}$} \\
\hline
\end{tabular}

fonction respiratoire, une nutrition entérale (par sonde nasogastrique ou gastro-entérostomie) peut être nécessaire afin de normaliser les paramètres anthropométriques [26]. Des formules riches en énergie $(1 \mathrm{kcal} / \mathrm{ml})$ pour nutrition entérale peuvent être administrées de préférence pendant la nuit afin de ne pas trop interférer avec les prises habituelles d'aliments et la vie quotidienne. Une supplémentation enzymatique avant le coucher et le début de la perfusion est habituellement suffisante afin d'obtenir une absorption satisfaisante. Ce traitement est souvent nécessaire chez les patients en attente d'une transplantation, mais pourrait être même plus efficace s'il était administré précocement en raison de troubles de l'alimentation, situation où cette méthode peut être temporaire. Une nutrition parentérale totale n'est habituellement pas indiquée.

\section{Supplémentation en vitamines}

La difficulté de la normalisation de l'absorption des lipides nécessite une supplémentation en vitamines liposolubles. Les capacités oxydatives étant accrues chez les patients atteints d'une mucoviscidose, pour des raisons inconnues [27], une attention particulière doit être portée à des antioxydants tels que la vitamine $\mathrm{E}$ et le $\beta$-carotène, dont les concentrations sont habituellement très basses dans la mucoviscidose. La vitamine A est importante pour l'intégrité des muqueuses, mais doit faire l'objet d'une attention particulière après une transplantation, moment où ses concentrations sériques deviennent souvent élevées. Les concentrations sériques des vitamines liposolubles doivent être régulièrement vérifiées, au moins une fois par an, particulièrement parce qu'elles pourraient être toxiques si elles sont trop élevées. Pour 
une analyse, voir de récentes déclarations de consensus résumant les pratiques nutritionnelles $[28,29]$. Le tableau 1 résume les recommandations concernant le traitement nutritionnel.

\section{Prise en charge des complications intestinales}

Une anorexie est parfois liée à des douleurs abdominales récurrentes dues à des complications intestinales telles qu'une sténose ou un syndrome d'occlusion intestinale distale. La première doit être traitée par la chirurgie mais la seconde peut habituellement céder à un lavement contenant $10 \%$ de $\mathrm{N}$-acétylcystéine et un produit de contraste radiologique hyperosmolaire dans un rapport 1/1 ou 2/1; la dose de $N$-acétylcystéine ne doit pas excéder 300 $\mathrm{mg} / \mathrm{kg}$. Chez les patients dont les troubles récidivent fréquemment, la prise orale d'importantes quantités quotidiennes de polymères inertes, tels qu'un mélange de polyéthylènes glycol de différents poids moléculaires, associée à l'éviction des aliments riches en fibres, peut avoir une certaine efficacité [30].

\section{Traitement de l'atteinte respiratoire}

Le traitement des troubles respiratoires a fait l'objet de la plus grande attention, car l'atteinte respiratoire est responsable de $95 \%$ de la mortalité due à la mucoviscidose. Bien que toutes les stratégies s'appuient sur une association de mucolytiques, d'activités physiques passives ou actives et d'antibiotiques, leur succès varie fortement tant entre pays qu'entre centres [31]. La colonisation chronique par Pseudomonas aeruginosa a très fortement retenu l'attention, bien que la colonisation plus ou moins chronique par Staphylococcus aureus, qui précède habituellement la première, induise une exacerbation respiratoire beaucoup plus intense, et donc également plus agressive pour les tissus [32]. Le risque existe que la crainte d'une colonisation par Pseudomonas, qui nécessite une antibiothérapie intraveineuse, conduise à négliger inconsciemment le traitement d'une infection à S. aureus. Les espèces de $P$. aeruginosa sont des bactéries aérobies facultatives très répandues dans la nature, et les patients intensivement traités pour des troubles discrets peuvent en être porteurs pendant des décennies sans détérioration de leur fonction respiratoire [33]. D’autres bactéries sont fréquentes dans la mucoviscidose comme Haemophilus influenzae et Stenotrophomonas maltophilia. Le complexe Burkholderia cepacia est un groupe de bactéries dont certaines souches sont responsables d'une évolution sévère et contagieuse, souvent appelée «syndrome cepacia».

Dépistage, thérapie et évolution clinique de la muscoviscidose
Des antibiothérapies intensives à long terme, mises au point par de nombreux centres, ont entraîné des problèmes de résistances bactériennes. La crainte de la dissémination de ces souches entre patients a conduit à séparer les unités d'hospitalisation des patients sur la base de la colonisation bactérienne, ce qui a provoqué un important stress psychologique pour ceux-ci $[34,35]$. Une récente analyse Cochrane pose la questions de savoir si une charge antibiotique élevée, qui serait instituée pour que $P$. aeruginosa ne soit identifié que de façon intermittente et non chronique, est justifiée à la lumière des problèmes que posent les souches résistantes et l'isolement des patients [36]. Une récente enquête sur les politiques de traitement dans des pays nordiques a montré que certains centres n'appliquent pas cette politique et que la prévalence de Pseudomonas est comparativement plus élevée chez leurs patients, mais avec moins de problèmes posés par des souches résistantes [37]. Les paramètres anthropométriques et la fonction respiratoire sont satisfaisants chez ces patients et leur qualité de vie l'est donc également, ce qui indique que des stratégies thérapeutiques alternatives pourraient être intéressantes à envisager [Lindblad A et coll., pour le Scandinavian CF Consortium, à paraître] (fig. 1).

Le tableau 2 mentionne différents agents fréquemment administrés pour le traitement de l'atteinte respiratoire. Différentes stratégies thérapeutiques ont été mises au point, la plupart centrées sur la prévention d'une colonisation chronique par des bactéries à Gram négatif, essentiellement $P$. aeruginosa. Ces stratégies comprennent l'administration généralisée de mucolytiques, une kinésithérapie et des antibiotiques. Le mucus très collant adhère à la muqueuse et, à titre de substrat, contribue à la colonisation bactérienne (fig. 2). Au moment du diagnostic, et afin de prévenir cette évolution, la plupart des centres instaurent un traitement par mucolytique seul jusqu'à l'isolement d'une bactérie. Ce traitement peut comporter des activités physiques [38] et inclure, à un stade précoce, une kinésithérapie sur ballon et trampoline. Dans certains pays, une kinésithérapie quotidienne plus passive avec techniques de percussion («clapping») est encore très utilisée. A partir du diagnostic, l'administration orale de bromhexine prévient la formation de bouchons de mucus collant et réduit les manifestations de sinusite chronique. L'inhalation quotidienne de sérum physiologique, d'un bronchodilatateur et d'un mucolytique est également introduite précocement; la fréquence est habituellement déterminée par la sévérité des symptômes. Il est établi que tous ces médicaments augmentent l'expectoration et réduisent les troubles respiratoires, mais leur

Ann Nestlé [Fr] 2006;64:131-141 
Tableau 2. Stratégies de traitement respiratoire les plus souvent utilisées pour les mucolytiques et les antibiotiques ${ }^{1}$

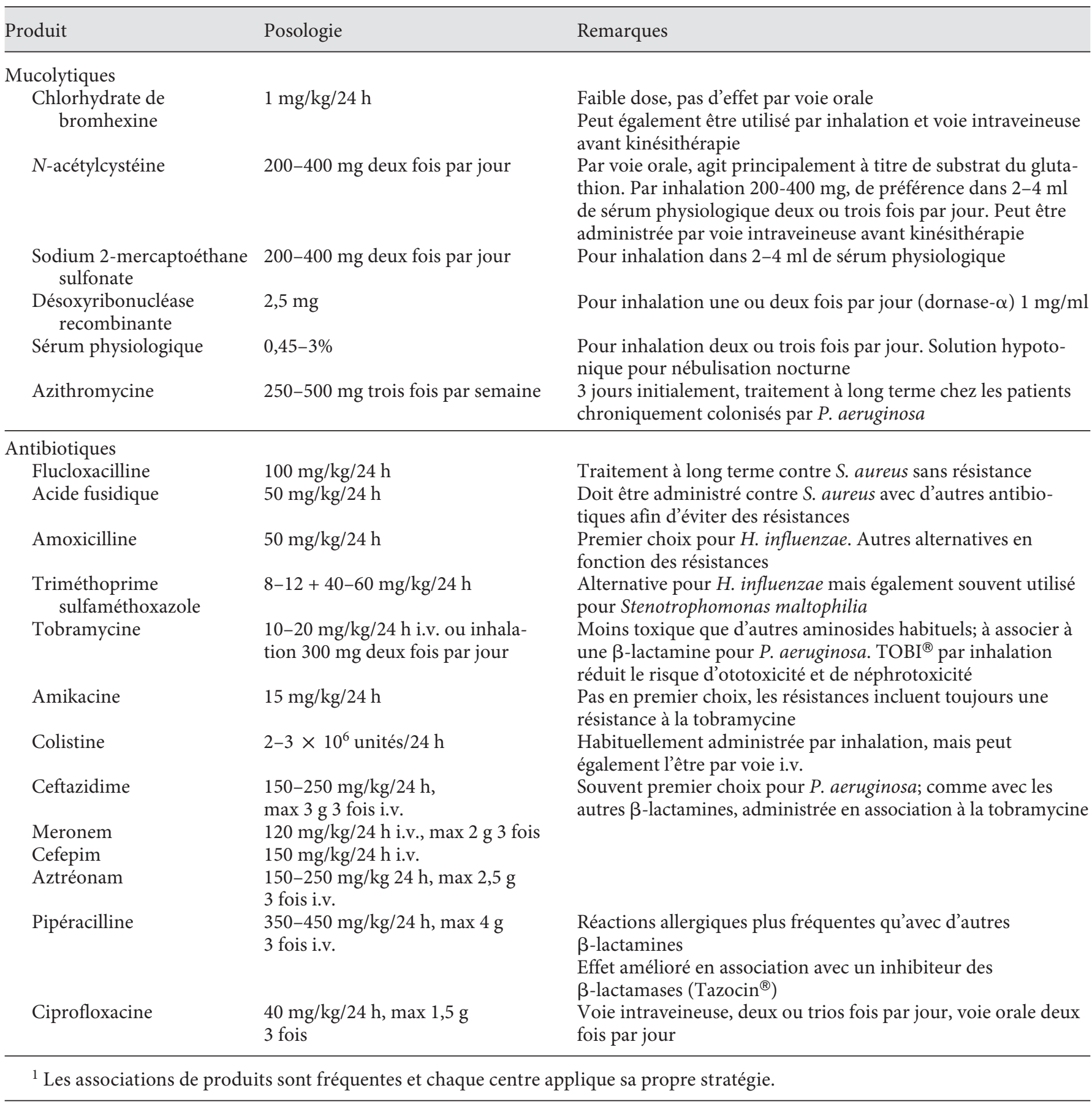

rôle préventif est moins net. D’un point de vue clinique, le choix des médicaments doit être individualisé: chez certains patients, le meilleur effet est obtenu avec la dornase- $\alpha$ et chez d'autres, il est aussi bon avec du sérum physiologique uniquement $[39,40]$. Avec tous les traite- ments inhalés, l'activité physique et/ou la kinésithérapie, également individualisées en fonction de l'observance, donne les meilleurs résultats pour l'expectoration. Ces dernières années, l'azithromycine a été administrée à titre de mucolytique. Son effet est remarquable en cas de 


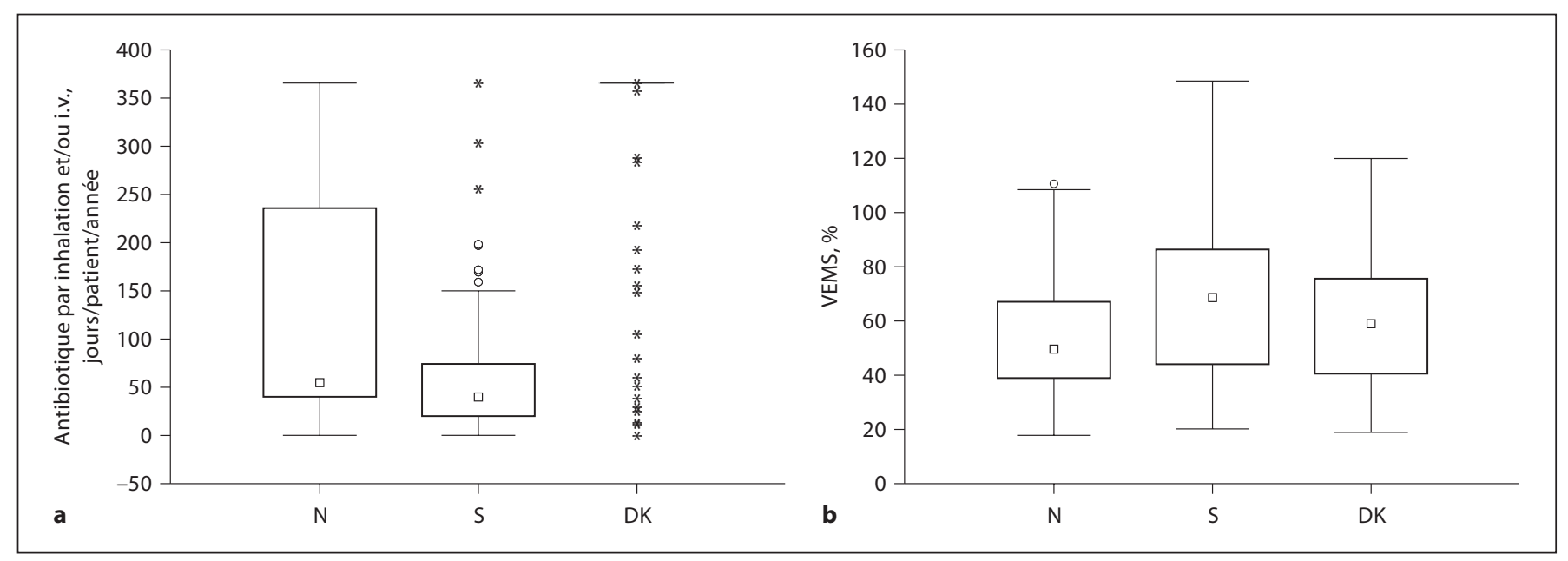

Fig. 1. Graphe à boîtes du traitement anti-Pseudomonas chez des patients âgés de $>19$ ans colonisés de façon chronique par $P$. aeruginosa dans trois pays nordiques: Norvège $(\mathrm{N})$, Suède $(\mathrm{S})$ et Danemark (DK; a) et fonction respiratoire correspondante exprimée par le volume maximal expiratoire par seconde (VEMS) en pourcentage de la valeur prévue (b). a Nombre de jours avec antibio- thérapie par inhalation et/ou intraveineuse par patient et année. Les médianes et les 25 et $75^{\text {ème }}$ percentiles sont indiqués. Les «moustaches» indiquent les 5 et $95^{\text {ème }}$ percentiles, les points les valeurs aberrantes et les astérisques les valeurs extrêmes. Reproduit avec autorisation du Scandinavian CF Consortium, Docteur A. Lindblad.

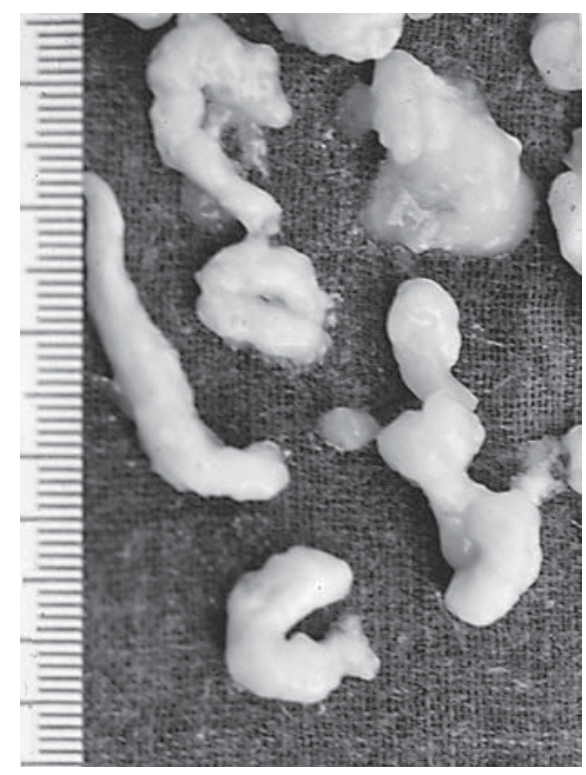

Fig. 2. Bouchons de mucus obtenus par bronchoscopie chez un enfant atteint de mucoviscidose. Photographie: Docteur Jean Feigelson, Paris, avec autorisation.

colonisation chronique par $P$. aeruginosa, bien que cette bactérie ne soit pas sensible à cet antibiotique. Une administration à long terme a pu réduire le nombre de traitements par antibiotiques intraveineux.

Dépistage, thérapie et évolution clinique de la muscoviscidose

\section{Stratégies antibiotiques}

En règle générale, une infection à $S$. aureus est traitée dès l'isolement de cette bactérie, et l'effet du traitement peut être facilement suivi par la mesure d'anticorps sériques spécifiques, tels ceux dirigés contre l'acide teichoïque et l' $\alpha$-toxine [41]. Des échantillons d'expectoration pouvant être difficiles à obtenir chez les jeunes enfants, certains centres administrent un traitement préventif à long terme par l'acide fusidique ou la flucloxacilline au cours de la petite enfance, tandis que d'autres s'en abstiennent en raison de la crainte d'une accélération de la colonisation par des bactéries Gram négatif. Il est important de noter qu'une résistance à la flucloxacilline est extrêmement rare et que l'isolement de souches méthicillinorésistantes est encore très peu fréquent dans des pays comme la Suède, où cet antibiotique est administré à long terme [37].

Un traitement intensif est la règle de nos jours lors du premier isolement de $P$. aeruginosa, ce qui peut permettre de retarder une colonisation chronique. La plupart des centres administrent une antibiothérapie intraveineuse associant un aminoside, habituellement la tobramycine, à une $\beta$-lactamine, suivie d'inhalations de tobramycine ou de colistine pendant plusieurs semaines ou mois [42]. Certains centres instaurent des inhalations régulières de tobramycine $\left(\mathrm{TOBI}^{\circledR}\right)$ pendant 4 semaines tous les 2 mois. D’autres utilisent un schéma similaire pour des inhala- 
tions de colistine. Le risque de colonisation chronique (défini comme plus d'un isolement de $P$. aeruginosa en 6 mois et un titre positif d'anticorps anti-Pseudomonas [43]) est d'autant plus faible que le traitement est plus intensif et prolongé alors qu'une colonisation intermittente est la conséquence d'une consommation élevée d'antibiotiques. Cette charge élevée en antibiotiques résulte également en une colonisation chronique par d'autres types de bactéries telles que Achromobacter xylosoxidans. Comme le montre la figure 1, dans les pays nordiques, où la charge antibiotique est la plus basse, la fonction respiratoire des patients est tout aussi bonne que dans d'autres pays, ce qui indique que la stratégie d'utilisation des antibiotiques est d'une grande importance. Chez certains patients atteints d'un infection sévère par une souche de Pseudomonas de sensibilité réduite, une perfusion continue d'une $\beta$-lactamine peut être effectuée avec des effets satisfaisants. En règle générale, le risque de survenue de résistances est moindre si le traitement est maintenu à 7-14 jours (moyenne 10). Si un traitement plus long est nécessaire, les antibiotiques ne doivent pas être modifiés $[33,37]$. Selon notre expérience, il est moins difficile d'éradiquer les souches mucoïdes que les souches nonmucoïdes. La raison n'en est pas évidente, mais pourrait être liée à la stratégie d'utilisation des mucolytiques et des antibiotiques.

Une antibiothérapie intraveineuse dès la constatation de signes d'exacerbation des troubles respiratoires est la modalité thérapeutique la plus largement utilisée. Dans la lutte destinée à maintenir la charge infectieuse aussi basse que possible, un traitement plus ou moins continu par inhalation a été plus fréquent ces dernières années. Le développement d'une formulation spéciale de tobramycine pour inhalation $\left(\mathrm{TOBI}^{\circledR}\right)$ a modifié la politique de traitement dans de nombreux pays, qui est passé d'une administration intraveineuse à une antibiothérapie à long terme pendant 4 semaines tous les 2 mois, traitement qui a amélioré la fonction respiratoire et l'a maintenue relativement stable pendant des durées prolongées [44]. Des résultats similaires peuvent être obtenus avec un traitement traditionnel reposant sur l'association de la tobramycine et d'une $\beta$-lactamine, avec moins de problèmes concernant la survenue de résistances bactériennes [33,45], comme l'illustre le fait que les pays nordiques, où la consommation d'antibiotiques est la plus basse chez les patients mucoviscidosiques, utilisent rarement un traitement inhalé. Le coût de l'antibiothérapie intraveineuse peut être également maintenu relativement bas en enseignant aux parents et aux patients comment la réaliser à domicile, ce qui donne également à la famille plus de liberté et se traduit donc par une meilleure observance et une meilleure acceptation du traitement [46]. Les prélèvements sanguins, qui sont nécessaires au cours de chaque cycle après la troisième dose d'aminoside pour contrôler la concentration sanguine et effectuer des corrections dans le but d'éviter une toxicité, peuvent être réalisés à domicile et adressés à l'hôpital.

Un problème survient parfois avec l'apparition d'une réaction allergique à l'antibiotique préférentiel, ou même au seul auquel la bactérie est sensible selon l'antibiogramme. Ce problème peut être surmonté par une désensibilisation très soigneuse, en débutant par de très basses concentrations de l'antibiotique au cours du traitement [47]. De plus longues périodes de traitement peuvent être nécessaires, particulièrement chez les patients sévèrement atteints attendant une transplantation pulmonaire, et une diversification des antibiotiques est alors importante.

\section{Corticothérapie ou non?}

Une corticothérapie est parfois administrée afin de réduire l'inflammation, mais son administration systémique accroît le risque de diabète clinique et doit donc être utilisée de façon restrictive. L'administration de nouvelles formulations de corticoïdes inhalés s'est accrue, mais il n'existe encore aucune étude montrant que l'effet antiinflammatoire des corticoïdes est plus efficace que l'antibiothérapie [48].

\section{Traitement de l'hypoxie}

L'oxymétrie de pouls doit être régulièrement vérifiée, car l'oxygénation peut diminuer de façon insidieuse, même quand la fonction respiratoire semble bien préservée. Le mécanisme sous-jacent est mal élucidé. Le premier signe apparaît habituellement la nuit au cours d'une exacerbation respiratoire [49] et, bien que ce fait soit largement reconnu, il n'existe aucune étude montrant comment il serait possible de mieux la prendre en charge dans la mucoviscidose [50]. Une supplémentation nocturne en oxygène est souvent recommandée chez les patients présentant une altération modérée de l'oxygénation, mais, à un certain degré, une oxygénothérapie continue devient nécessaire. Dans l'attente d'une transplantation, un traitement par ventilation contrôlée en pression peut être utile, et peut également être un complément nécessaire en cas d'exacerbation sévère [51]. La ventilation mécanique est contre-indiquée car il peut être impossible d'extuber le patient. 


\section{Indication d'une transplantation pulmonaire}

Une transplantation pulmonaire doit être envisagée quand la fonction respiratoire du patient est altérée avec un VEMS $<30 \%$ de la valeur prévue. La décision de placer le patient sur liste d'attente pour transplantation pulmonaire doit prendre en compte de nombreux facteurs tels que l'état nutritionnel, la vitesse de déclin de la fonction respiratoire, le type de colonisation bactérienne, des facteurs psychologiques, etc. [52].

\section{Dépistage}

Le dépistage de la mucoviscidose a été introduit dans les années 60 en utilisant la détermination de l'albumine fécale à titre d'indicateur d'une insuffisance pancréatique. Cet examen n'a jamais été largement utilisé en raison des problèmes majeurs que posaient des résultats tant faussement positifs que négatifs. Une augmentation de la trypsine immunoréactive dans le sérum s'est avérée d'une spécificité et d'une sensibilité plus élevées et a été récemment utilisée à titre de premier moyen, parfois répété et habituellement suivi par une analyse des mutations régionales les plus fréquentes, avant la réalisation d'un test de la sueur [53]. L'objectif de l'introduction du dépistage est d'éviter la survenue de lésions respiratoires, et les résultats à court terme ont été positifs tant en termes de nutrition que d'atteinte respiratoire [54], mais, après l'âge de 10 ans, la plupart des études ont montré très peu de différence par rapport aux patients non dépistés [55]. Ce résultat a été décevant et la déclaration la plus récente de consensus ne recommande généralement pas le dépistage dans la population générale mais le considère comme justifié dans certaines régions et à des fins particulières [53]. De nombreuses études très détaillées de suivi de populations dépistées ont amélioré les connaissances sur l'histoire naturelle des manifestations précoces de la maladie. Les problèmes liés au dépistage sont les suivants: diagnostic des cas asymptomatiques ou ne présentant des troubles précoces que très discrets, diagnostic des porteurs sains, perturbation précoce du lien mère-nourrisson, inaptitude à identifier des troubles discrets chez des patients non identifiés par le dépistage, et coûts [55]. Il n'existe encore aucun traitement curatif de la mucoviscidose, et l'introduction du dépistage doit donc être envisagée à l'échelon régional, en étroite coopération avec les centres de traitement de la mucoviscidose, et en mettant en balance les coûts et les bénéfices tant pour les sujets que pour la société.

Dépistage, thérapie et évolution clinique de la muscoviscidose

\section{Évolution clinique}

La mucoviscidose n'est plus une maladie atteignant uniquement des enfants. Dans un nombre croissant de centres, $50 \%$ des patients, ou environ, sont des adultes. La survie médiane a augmenté jusqu'au moins le milieu de la troisième décennie tant aux États-Unis qu'en Europe de l'Ouest, et est même plus longue dans certains centres [56]. Il existe toutefois d'importantes différences régionales dans le restant du monde, essentiellement dues à des facteurs socio-économiques; par exemple, dans certains pays de l'ancienne Europe de l'Est, l'absence des traitements médicaux les plus fondamentaux ne laisse aucune option thérapeutique. Dans les pays industrialisés, la plupart des patients bénéficient aujourd'hui d'une vie sociale satisfaisante et parviennent à trouver un emploi à temps complet ou partiel. De plus, les hommes atteints d'une mucoviscidose peuvent fonder une famille à la suite d'une aspiration réussie de sperme suivie d'une fécondation in vitro. Bien que l'incidence du diabète lié à la mucoviscidose augmente avec l'âge, ce type de diabète est habituellement de traitement facile et son diagnostic précoce ainsi qu'un traitement par insuline rapide peut prévenir l'aggravation souvent décrite de cette complication. La présence d'une hépatopathie n'influence quasiment jamais la survie du patient. Elle ne semble généralement pas progresser avec l'âge et, si elle progresse, le résultat d'une transplantation hépatique est à présent généralement bon, souvent également avec une amélioration des manifestations respiratoires [57]. Une transplantation simultanée de poumon et de foie est donc rarement indiquée, mais des transplantations simultanées du pancréas et du foie se sont récemment avérées efficaces [58]. La longévité est encore prolongée par la réussite d'une transplantation pulmonaire. Le taux de survie à 5 ans des patients atteints d'une mucoviscidose est généralement plus élevé que celui des patients transplantés en raison d'autres maladies, probablement parce qu'ils sont plus jeunes et particulièrement s'ils sont également en bon état nutritionnel $[59,60]$.

\section{Conclusion}

Plus de 16 ans se sont maintenant écoulés depuis la découverte de l'anomalie génétique sous-tendant la mucoviscidose et, sur cette base, les connaissances ont fortement progressé à la suite d'une intensification remarquable des recherches fondamentales. Plusieurs innovations potentielles ont été réalisées pour le traitement de fond de

Ann Nestlé [Fr] 2006;64:131-141 
la maladie, mais, à ce jour, la forte prolongation de la survie est due au développement continu du traitement traditionnel, à des soins centralisés et aux données indiquant que le suivi fréquent du patient peut permettre d'identifier des manifestations respiratoires discrètes. Un traitement intensif préviendra la survenue de lésions pulmonaires progressives. Dans ce contexte, une observa- tion particulière est nécessaire afin qu'un traitement à long terme par inhalation d'antibiotiques et de plus rares visites au centre clinique ne s'opposent pas à l'intention du traitement. Il est difficile, pour le centre comme pour l'équipe, de motiver les politiciens afin qu'ils accordent des ressources destinées aux traitements et options adéquats, dans le plus grand intérêt des patients.

\section{Références}

1 Fanconi G, Uehlinger E, Knauer C: Das Coeliakiesyndrom bei angeborenen zystischer Pancreasfibromatose und Bronchiektasien. Wien Med Wochenschr 1936;86:753-756.

2 Andersen DH: Cystic fibrosis of the pancreas and its relation to celiac disease: a clinical and pathological study. Am J Dis Child 1938; 56:344-399.

3 Bodian M: Fibrocystic Disease of the Pancreas: A Congenital Disorder of Mucus Production - Mucosis. New York, Grune \& Stratton, 1953.

-4 Marks BL, Anderson CM: Fibrocystic disease of the pancreas in a man aged 46. Lancet 1960;1:365.

5 van Koolwijk LME, Uiterwaal CSPM, van der Laag J, et al: Treatment of children with cystic fibrosis: central, local or both? Acta Paediatr 2002;91:972-977.

6 Littlewood JM: Good care for people with cystic fibrosis. Paediatr Respir Rev 2000;1: 179-189.

7 Thomson MA, Wilmott RW, Wainwright C, et al: Resting energy expenditure, pulmonary inflammation, and genotype in the early course of cystic fibrosis. J Pediatr 1996; 129:367-373.

8 O’Rawe A, McIntosh I, Dodge JA, et al: Increased energy expenditure in cystic fibrosis is associated with specific mutations. Clin Sci 1992;82:71-76.

$\checkmark 9$ Fried MD, Durie PR, Tsui L-C, et al: The cystic fibrosis gene and resting energy expenditure. J Pediatr 1991;119:913-916.

10 Kindstedt-Arfwidson K, Strandvik B: Food intake in patients with cystic fibrosis on an ordinary diet. Scand J Gastroenterol 1988; 23:160-162.

11 Kawchak DA, Zhao H, Scanlin TF, et al: Longitudinal, prospective analysis of dietary intake in children with cystic fibrosis. J Pediatr 1996;129:119-129.

12 Strandvik B, Gronowitz E, Enlund F, et al: Essential fatty acid deficiency in relation to genotype in patients with cystic fibrosis. J Pediatr 2001;139:650-655.

13 Farrell PM, Mischler EH, Engle MJ, et al: Fatty acid abnormalities in cystic fibrosis. Pediatr Res 1985;19:104-109.
14 Kurlandsky LE, Bennink MR, Webb PM, et al: The absorption and effect of dietary supplementation with omega- 3 fatty acids on serum leukotriene $\mathrm{B}_{4}$ in patients with cystic fibrosis. Pediatr Pulmonol 1994;18:211-217.

15 Strandvik B, Svensson E, Seyberth HW: Prostanoid biosynthesis in patients with cystic fibrosis. Prostagland Leukotr Essent Fatty Acids 1996;55:419-425.

16 Stark LJ: Can nutrition counselling be more behavioural? Lessons learned from dietary management of cystic fibrosis. Proc Nutr Soc 2003;62:793-799.

17 Baker SS, Borowitz D, Duffy L, et al: Pancreatic enzyme therapy and clinical outcomes in patients with cystic fibrosis. J Pediatr 2005;146:189-193.

18 Strandvik B, Hansson L, Hernell O, et al: Recombinant human bile salt-stimulated lipase improves lipid uptake and reduces the pancreatic enzyme supplementation in patients with cystic fibrosis. Pediatr Pulmonol 2004(suppl 27):333.

19 Borowitz D, Goss CH, Stevens C, et al: Safety and preliminary clinical activity of a novel pancreatic enzyme preparation in pancreatic insufficient cystic fibrosis patients. Pancreas 2006;32:258-263.

20 Strandvik B, Einarsson K, Lindblad A, Angelin B: Bile acid kinetics and biliary lipid composition in cystic fibrosis. J Hepatol 1996;25: 43-48.

21 Harries JT, Muller DPR, McCollum JPK, et al: Intestinal bile salts in cystic fibrosis. Arch Dis Child 1979;54:19-24.

22 Perin N, Jarocka-Cyrta E, Keelan M, et al: Dietary lipid composition modifies intestinal morphology and nutrient transport in young rats. J Pediatr Gastroenterol Nutr 1999;28:46-53.

23 Maqbool A, Schall J, Zemel B, et al: Essential fatty acid deficiency and the relevance of the triene:tetraene ratio in children with cystic fibrosis. J Pediatr Gastroenterol Nutr 2005; 41:550.

24 Strandvik B, Berg U, Kallner A, Kusoffsky E: Effect on renal function of essential fatty acid supplementation in cystic fibrosis. J Pediatr 1989;115:242-250.
25 Strandvik B, Hultcrantz R: Liver function and morphology during long term fatty acid supplementation in cystic fibrosis. Liver 1994;14:32-36.

26 van Biervliet S, De Waele K, van Winckel M, Robberecht E: Percutaneous endoscopic gastrostomy in cystic fibrosis: patient acceptance and effect of overnight tube feeding on nutritional status. Acta Gastroenterol Belg 2004;67:241-244.

27 Brown RK, Kelly FJ: Evidence for increased oxidative damage in patients with cystic fibrosis. Pediatr Res 1994;36:487-493.

28 Sinaasappel M, Stern M, Littlewood J, et al: Nutrition in patients with cystic fibrosis: a European Consensus. J Cyst Fibr 2002;1:5175.

29 Borowitz D, Baker RD, Stallings V: Consensus report on nutrition for pediatric patients with cystic fibrosis. J Pediatr Gastroenterol Nutr 2002;35:246-259.

30 Proesmans M, De Boeck K: Evaluation of dietary fiber intake in Belgian children with cystic fibrosis: Is there a link with gastrointestinal complaints? J Pediatr Gastroenterol Nutr 2002;35:610-614.

31 Ferkol T, Rosenfeld M, Milla CE: Cystic fibrosis pulmonary exacerbations. J Pediatr 2006;148:259-264.

32 Hollsing AE, Lantz B, Bergström K, et al: Granulocyte elastase-alpha1-antiproteinase complex in cystic fibrosis: sensitive plasma assay for monitoring pulmonary infections. J Pediatr 1987;111:206-211.

33 Strandvik B: Antibiotic therapy of pulmonary infections in cystic fibrosis. Dosage and dose schedules. Chest 1988;94:146S-149S.

34 Koch C, Frederiksen B, Hoiby N: Patient cohorting and infection control. Semin Respir Crit Care Med 2003;24:703-716.

35 Steinkamp G, Ullrich G: Different opinions of physicians on the importance of measures to prevent acquisition of Pseudomonas aeruginosa from the environment. J Cyst Fibros 2003;2:199-205.

36 Wood DM, Smyth AR: Antibiotic strategies for eradicating Pseudomonas aeruginosa in people with cystic fibrosis. The Cochrane Database of Systematic Reviews 2006;DOI: 10.1002/14651858.CD004197.pub2. 
37 Strandvik B, Hjelte L, Malmborg AS: Cystic fibrosis pulmonary infection: the Swedish experience; in Bauernfeind A, Marks MI, Strandvik B (eds): Cystic Fibrosis Pulmonary Infections: Lessons from Around the World. Basel, Birkhäuser, 1996, pp 293-302.

- 38 Blomquist M, Freyschuss U, Wiman LG, Strandvik B: Physical activity and self treatment in cystic fibrosis. Arch Dis Child 1986; 61:362-367.

39 Suri R: The use of human deoxyribonuclease (rhDNase) in the management of cystic fibrosis. BioDrugs 2005;19:135-144.

-40 Elkins MR, Robinson M, Rose BR, et al: A controlled trial of long-term inhaled hypertonic saline in patients with cystic fibrosis. $\mathrm{N}$ Engl J Med 2006;354:229-240.

41 Hollsing AE, Granström M, Strandvik B: Prospective study of serum staphyloccocal antibodies in cystic fibrosis. Arch Dis Child 1987;62:905-911.

42 Cantón R, Cobos N, de Gracia J, et al; on behalf of the Spanish Consensus Group for Antimicrobial Therapy in the Cystic Fibrosis Patient: Antimicrobial therapy for pulmonary pathogenic colonisation and infection by Pseudomonas aeruginosa in cystic fibrosis patients. Clin Microbiol Infect 2005;11:690-703.

-43 Ericsson-Hollsing A, Granström M, Vasil M, et al: Prospective study of serum antibodies to Pseudomonas aeruginosa exoproteins in cystic fibrosis. J Clin Microbiol 1987;25: 1868-1874.
44 Cheer SM, Waugh J, Noble S: Inhaled tobramycin (TOBI): a review of its use in the management of Pseudomonas aeruginosa infections in patients with cystic fibrosis. Drugs 2003;63:2501-2520.

45 Blumer JL, Saiman L, Konstan MW, Melnick $D$ : The efficacy and safety of meropenem and tobramycin vs ceftazidime and tobramycin in the treatment of acute pulmonary exacerbations in patients with cystic fibrosis. Chest 2005;128:2336-2346.

46 Strandvik B, Hjelte L, Malmborg AS, Widén B: Home intravenous antibiotic treatment of patients with cystic fibrosis. Acta Paediatr Scand 1992;81:340-344.

47 Parmar JS, Nasser S: Antibiotic allergy in cystic fibrosis. Thorax 2005;60:517-520.

48 Dezateux C, Walters S, Balfour-Lynn I: Inhaled corticosteroids for cystic fibrosis. The Cochrane Database Systematic Reviews 2000;2:CD001915.

49 Villa MP, Pagani J, Lucidi V, et al: Nocturnal oximetry in infants with cystic fibrosis. Arch Dis Child 2001;84:50-54.

50 Mallory GB, Fullmer JJ, Vaughan DJ: Oxygen therapy for cystic fibrosis. The Cochrane Database of Systematic Reviews 2005;4. DOI: 10.002/14651858. CD003884.pub2.

51 Dobbin CJ, Milross MA, Piper AJ, et al: Sequential use of oxygen and bi-level ventilation for respiratory failure in cystic fibrosis. J Cyst Fibros 2004;3:237-242.

-52 Rosenbluth DB, Wilson K, Ferkol T, Schuster DP: Lung function decline in cystic fibrosis patients and timing for lung transplantation referral. Chest 2004;126:412-419.
53 Grosse SD, Boyle CA, Botkin JR, et al: CDC: Newborn screening for cystic fibrosis: evalutation of benefits and risks and recommendations for state newborn screening programs. MMWR Recomm Rep 2004;53:136.

54 Dankert-Roelse JE, Mérelle ME: Review of outcomes of neonatal screening for cystic fibrosis versus non-screening in Europe. J Pediatr 2005;147:S15-S20.

55 Castellani C: Evidence for newborn screening for cystic fibrosis. Paediatr Respir Rev 2003;4:278-284.

56 Lannefors L, Lindgren A: Demographic transition of the Swedish cystic fibrosis community - results of modern care. Respir Med 2002;96:681-685.

57 Meyburg J, Hoffmann GF: Liver transplan tation for inborn errors of metabolism. Transplantation 2005;80:S135-S137.

58 Fridell JA, Vianna R, Kwo PY, et al: Simultaneous liver and pancreas transplantation in patients with cystic fibrosis. Transplant Proc 2005;37:3567-3569.

59 Bech B, Pressler T, Iversen M, et al: Longterm outcome of lung transplantation for cystic fibrosis - Danish results. Eur J Cardiol Thorac Surg 2004;26:1180-1186.

60 Ganesh JS, Rogers CA, Bonser RS, Banner NR: Outcome of heart-lung and bilateral sequential lung transplantation for cystic fibrosis: a UK national study. Eur Respir J 2005;25:964-969. 